\title{
Diversity and phylogeny of Neofusicoccum species occurring in forest and urban environments in Portugal
}

\author{
Lopes $\mathbf{A}^{1}$, Barradas $\mathbf{C}^{\mathbf{1}}$, Phillips $\mathrm{AJL}^{2}$ and Alves $\mathbf{A}^{\mathbf{1}}$

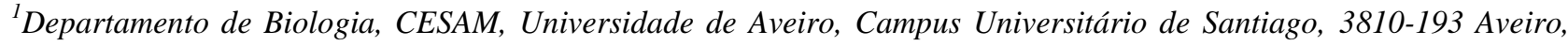 \\ Portugal \\ ${ }^{2}$ Biosystems and Integrative Sciences Institute, Faculty of Science, University of Lisbon, Campo Grande, 1749-016 \\ Lisbon, Portugal
}

Lopes A, Barradas C, Phillips AJL, Alves A 2016 - Diversity and phylogeny of Neofusicoccum species occurring in forest and urban environments in Portugal. Mycosphere 7(7), 906-920, Doi 10.5943/mycosphere/si/1b/10

\begin{abstract}
A collection of Neofusicoccum isolates was obtained from a large number of plant species, showing dieback and canker symptoms, in forest and urban environments in Portugal. A total of 351 isolates was characterised by BOX-PCR fingerprinting to evaluate their overall genetic diversity. Representatives of each group identified in this analysis were selected for multilocus sequence analyses, using sequences of the ribosomal internal transcribed spacer region (ITS rDNA) and partial sequences of the translation elongation factor 1-alpha (tef1) and $\beta$-tubulin (tub2). Phylogenetic analysis of multilocus sequence data identified five species within the collection of isolates, namely $N$. australe, $N$. eucalyptorum, $N$. kwambonambiense, $N$. luteum, and $N$. parvum. Of these $N$. australe and N. eucalyptorum were the most frequent representing the vast majority of the isolates. Several new fungus-host associations were established for all of the Neofusicoccum species found. Here we report for the first time the occurrence of $N$. eucalyptorum on a host outside the family Myrtaceae. The results of this study show that the genus Neofusicocccum appears to be common and widespread on a broad range of hosts representing a potential threat to susceptible plants. Additionally, ornamental plants in urban environments are shown to be hosts of a diverse assemblage of Neofusicoccum species.
\end{abstract}

Key words - Botryosphaeriaceae - endophytic - host-association - ornamentals - pathogenic

\section{Introduction}

The genus Neofusicoccum is a member of the Botryosphaeriaceae (Botryosphaeriales, Dothideomycetes) comprising numerous species found on a wide range of plant hosts of agricultural, forestry, ecological and economic importance (Crous et al. 2006, Slippers \&Wingfield 2007, Slippers et al. 2013). Host infections are thought to occur predominantly through horizontal transmission, i.e. individual infections via spores (ascospores or conidia) but also through the seeds (vertical transmission). Inside the plant, they have the ability to colonize without producing any external symptoms, remaining inside the host as endophytes (Slippers \& Wingfield 2007). Endophytism could be, however, an important feature since these fungi can be moved easily and inconspicuously around the world in seeds, cuttings and even fruits, subsequently infecting both native and non-native trees in their new environments (Burgess et al. 2005, Slippers et al. 2013). 
The change from endophytic to pathogenic phase is often related to stress such as drought, extreme temperature fluctuations, nutrient deficiencies and mechanical injuries. Infected plants can exhibit a multiplicity of disease symptoms such as fruit rots, leaf spots, seedling damping-off and collar rot, cankers, blight of shoots and seedlings, gummosis, blue-stain of the sapwood, dieback and tree death (Slippers \& Wingfield 2007).

Neofusicoccum is known to include a large number of phylogenetically closely related and morphologically similar cryptic species rendering phenotypic characteristics such as morphology, growth and culture appearance inadequate for species identification. Thus, species discrimination is based on a multilocus sequencing approach (Pavlic et al. 2009a, 2009b).

Within the 29 species currently accepted in the genus some are known to have very wide host and geographic ranges while others show some host preference. For example, $N$. parvum was reported from 90 hosts in 29 countries on six continents by Sakalidis et al. (2013). In contrast, $N$. eucalypticola and N. mangiferae were associated only with Eucalyptus spp. and Mangifera indica respectively and were geographically more restricted (Phillips et al. 2013). In general, species of Neofusicoccum are a constant presence in almost all kind of woody plants. For instance, they are frequently isolated from eucalypts (Iturritxa et al. 2011), almond (Inderbitzin et al. 2010), avocado (McDonald \& Eskalen 2011), walnut (Yu et al. 2015), grapevine (Mondello et al. 2013, BerrafTebbal et al. 2014), olive (Triki et al. 2015), blueberry (Pérez et al. 2014), mango (Ismail et al. 2013), rubber tree (Ngobisa et al. 2013) and peach (Thomidis et al. 2011). Although these fungi have been relatively well studied on economically important crops, much less is known about their prevalence in others groups of plants namely the gymnosperms (Slippers et al. 2005, Golzar \& Burgess 2011) and ornamental species (Zlatković et al. 2016). Although of low economic value, ornamental plant species should not be ignored as they provide a wide range of ecosystem services (Zlatković et al. 2016).

In Portugal the forest area occupies $35.4 \%$ of the total territory according to the National Forest Inventory 2013 (ICNF, 2013). Eucalyptus globulus, a non-native species, is the most abundant followed by Quercus suber and Pinus pinaster. Apart from the forest species Portugal has important crops such as grapevine and olive among others. In spite of this, knowledge about the diversity of the genus Neofusicoccum in Portugal is scarce. The few known studies were done on grapevines (Phillips 2002), conifers (Alves et al. 2013) and more recently on eucalypts (Barradas et al. 2016).

To gain further knowledge about the diversity of the genus Neofusicoccum in Portugal the main goal of this study was to identify the species associated with a wide diversity of plants. For this, a survey was performed on several species of forest and crop trees and also on ornamental species.

\section{Materials \& Methods}

\section{Fungal isolation and morphological characterization}

Isolates were obtained from samples collected from plants in natural forest ecosystems and ornamentals planted in urban environments (e.g. parks, gardens, streetscapes). The following hosts were sampled: Acacia longifolia, Aesculus hippocastanum, Castanea sativa, Ferula communis, Fraxinus angustifolia, Fraxinus excelsior, Fraxinus ornus, Hydrangea macrophylla, Malus domestica, Melia azedarach, Olea europaea, Populus alba, Populus tremula, Pyracantha coccinea, Quercus robur, Rosa sp., Tilia platyphyllos and Ulmus minor. This study also included isolates from diseased and healthy Eucalyptus globulus that were previously obtained by Barradas et al. (2016). The remaining isolates were obtained from hosts showing disease symptoms on stems, trunks and branches such as canker and dieback (Fig. 1). Samples from diseased plants were initially screened for the presence of fruiting bodies (ascomata and/or conidiomata) and when present single spore isolations were made as described previously (Phillips et al. 2013). In the absence of fruiting bodies isolations were made by plating pieces of diseased tissues following 


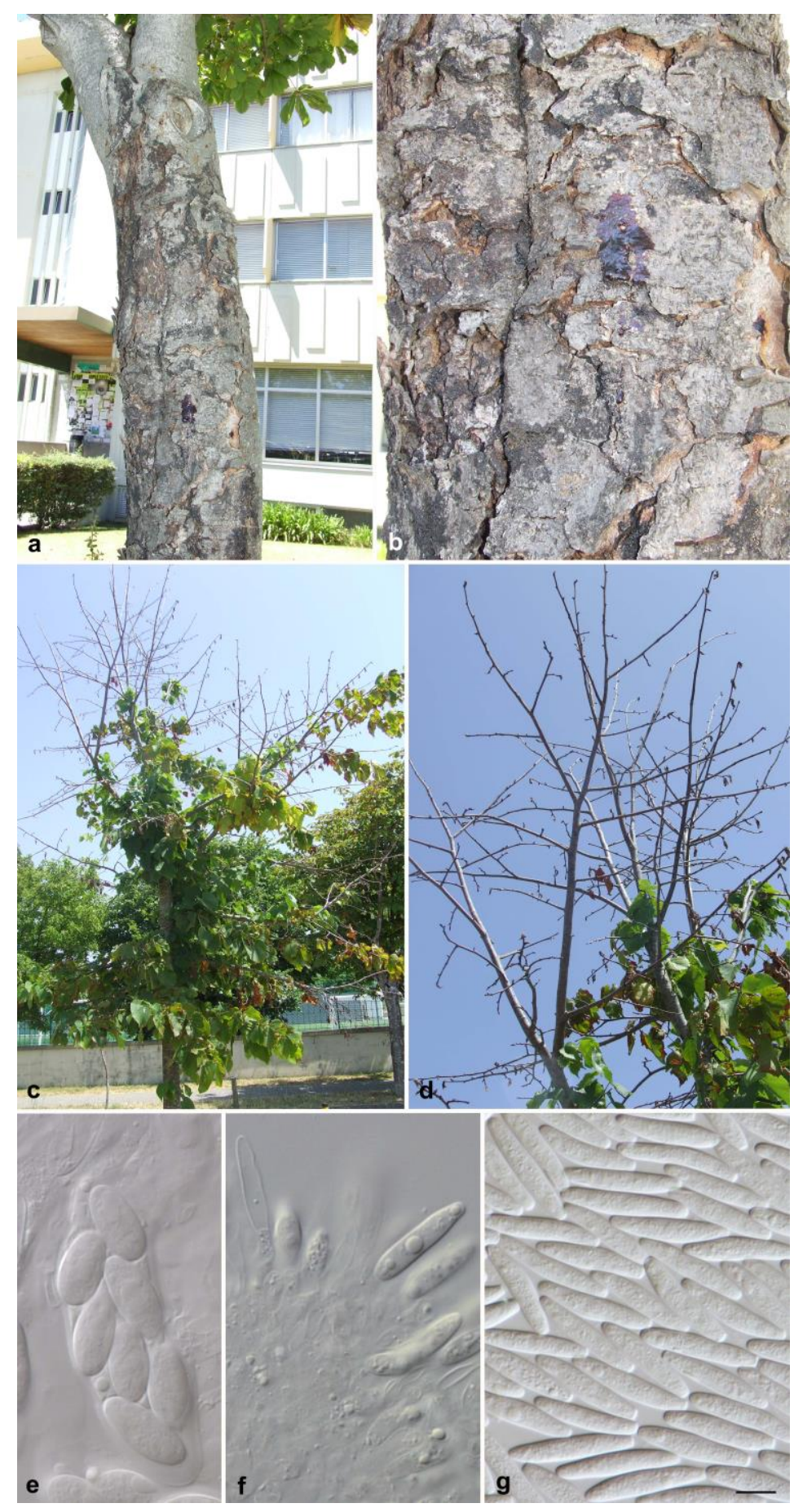

Fig. 1 - a. Aesculus hippocastanum with trunk canker. b. Detailed view of trunk canker. c,d. Tilia platyphyllos with symptoms of dieback of twigs and branches associated with $N$. luteum and $N$. australe. e. Ascus and ascospores of $N$. australe on Ferula communis. f. Developing conidia of $N$. luteum on Melia azedarach. g. Conidia of N. australe from Acacia longifolia.

surface sterilization as described by Alves et al. (2013). Cultures were routinely grown and maintained on half-strength potato-dextrose agar (PDA) (HIMEDIA, India).

To assign isolates to the genus Neofusicoccum conidial micromorphological characteristics and mode of conidiogenesis were observed with a Nikon 80i microscope and images captured with a Nikon DS-Ril camera. Isolates were induced to sporulate by plating them on $1 / 4$ strength PDA (Merck, Germany) containing sterilised pine needles and incubating at room temperature (about $20-25^{\circ} \mathrm{C}$ ) under diffused daylight until pycnidia developed. For microscopy, pycnidia were mounted in $100 \%$ lactic acid. 


\section{Molecular characterization}

Genomic DNA was extracted from fresh mycelium grown on half-strength PDA plates for 5 $\mathrm{d}$ at approximately $23^{\circ} \mathrm{C}$, according to Alves et al. (2004). All PCR reactions were carried out in 25 $\mu \mathrm{L}$ reaction mixtures with NZYTaq $2 \times$ Green Master Mix $(2.5 \mathrm{mM} \mathrm{MgCl} ; 200 \mu \mathrm{M}$ dNTPs; 0.2 $\mathrm{U} / \mu \mathrm{L}$ DNA polymerase) (Lisbon, Portugal) in a Bio-Rad C-1000 Touch $^{\mathrm{TM}}$ Thermal Cycler (Hercules, CA, USA). Negative controls with sterile water instead of the template DNA were used in every PCR reaction.

BOX-PCR fingerprinting was done as described previously (Alves et al. 2007) using primer BOXA1R. Representatives of each group identified in this analysis were selected for multilocus sequence analyses (Table 1). Primers ITS1 and ITS4 (White et al. 1990) were used for amplification and sequencing of the ITS region of the ribosomal RNA as described by Alves et al. (2004). Part of the translation elongation factor 1-alpha (tef1) was amplified with the primers EF1688F and EF1-1251R (Alves et al. 2008) with the conditions described by Phillips et al. (2005). Part of the $\beta$-tubulin gene (tub2) was amplified with primers T1 (O'Donnell \& Cigelnik 1997) and Bt2b (Glass \& Donaldson 1995) using the following conditions: $95^{\circ} \mathrm{C}$ for 3 min; 35 cycles at $94^{\circ} \mathrm{C}$ for $30 \mathrm{~s}, 50^{\circ} \mathrm{C}$ for $30 \mathrm{~s}$, and $72^{\circ} \mathrm{C}$ for $1 \mathrm{~min}$; final extension at $72^{\circ} \mathrm{C}$ for $10 \mathrm{~min}$.

PCR amplicons were purified with the DNA Clean \& Concentrator ${ }^{\mathrm{TM}^{-5}}$ kit (Zymo Research, CA, USA) before DNA sequencing. Both strands of the PCR products were sequenced at GATC Biotech (Cologne, Germany). The nucleotide sequences were read with FinchTV v.1.4.0 (Geospiza Inc.). All sequences were checked manually, and nucleotide arrangements at ambiguous positions were clarified using both primer direction sequences. Sequences were deposited in GenBank (Table 1) and the alignment in TreeBase (S19901).

Available ITS, tefl and tub2 sequences from known and well-characterized Neofusicoccum species were retrieved from GenBank and also included in the phylogenetic analyses. Sequences of Dothiorella sarmentorum and Do. iberica were used as outgroup (Table 1).

Sequences were aligned with ClustalX v. 2.1 (Thompson et al. 1997), using the following parameters: pairwise alignment parameters (gap opening $=10$, gap extension $=0.1$ ) and multiple alignment parameters (gap opening $=10$, gap extension $=0.2$, transition weight $=0.5$, delay divergent sequences $=25 \%$ ). Alignments were checked and edited with BioEdit Alignment Editor v. 7.2.5 (Hall 1999). Phylogenetic analyses of sequence data were done with MEGA6 v. 6.06 (Tamura et al. 2013). All gaps were included in the analyses. The model of DNA sequence evolution used for each dataset was determined by the software. Maximum likelihood (ML) analyses were performed on a neighbour-joining (NJ) starting tree automatically generated by the software. Bootstrap analyses with 1000 replicates were used to estimate the consistency of each node of the trees. Trees were visualized and edited with Interactive tree of life (iTOL) v3 (Letunic \& Bork 2016).

\section{Results}

\section{Fungal Isolation}

A collection of 351 isolates from different hosts was established. These isolates were initially selected based on culture characteristics typical of the Botryosphaeriaceae, namely fastgrowing fluffy white aerial mycelium becoming grey to black with grey to indigo-grey or black pigment visible from the reverse side of Petri dishes. Most isolates sporulated within 2-3 weeks on $1 / 4$ strength PDA supplemented with pine needles. Micromorphology of conidia and conidiogenesis assigned them to Neofusicoccum. Host ranges of the species were determined from the SMML Fungus-Host Distribution Database as well as available literature. Several new fungus-host associations were established (Table 2). 
Table 1 Identity of the isolates studied and GenBank accession numbers of sequences used in phylogenetic analyses.

\begin{tabular}{|c|c|c|c|c|c|c|}
\hline \multirow{2}{*}{ Species } & \multirow[b]{2}{*}{ Isolate $^{\text {a }}$} & \multirow[b]{2}{*}{ Origin } & \multirow[b]{2}{*}{ Host } & \multicolumn{3}{|c|}{ GenBank Accession $^{b}$} \\
\hline & & & & ITS & tef1 & $t u b 2$ \\
\hline Dothiorella iberica & CBS115041 & Spain & Quercus ilex & AY573202 & AY573222 & EU673096 \\
\hline D. sarmentorum & IMI63581b & United Kingdom & Ulmus sp. & AY573212 & AY573235 & EU673102 \\
\hline \multirow[t]{4}{*}{ N. algeriense } & CBS137504 & Algeria & Vitis vinifera & KJ657702 & KX505893 & KX505915 \\
\hline & CAA322 & Portugal & Malus domestica & KX505906 & KX505894 & $K X 505916$ \\
\hline & CAA366 & Portugal & Eucalyptus globulus & KT440951 & KT441011 & KX871764 \\
\hline & PE32 & Portugal & Eucalyptus globulus & KT440952 & KT441012 & KX871765 \\
\hline N. andinum & CBS117453 & Venezuela & Eucalyptus sp. & $G U 251155$ & $G U 251287$ & $G U 251815$ \\
\hline \multirow[t]{2}{*}{ N. arbuti } & CBS116131 & USA & Arbutus menziesii & AY819720 & KF531792 & KF531793 \\
\hline & CBS117090 & USA & Arbutus menziesii & AY819724 & KF531791 & KF531794 \\
\hline \multirow[t]{28}{*}{ N. australe } & CMW6837 & Australia & Acacia sp. & AY339262 & AY339270 & AY339254 \\
\hline & CMW6853 & Australia & Sequoiadendron giganteum & AY339263 & AY339271 & AY339255 \\
\hline & CAA178 & Portugal & Ferula communis & KX871844 & KX871800 & KX871709 \\
\hline & CAA184 & Portugal & Ferula communis & KX871845 & KX871801 & KX871710 \\
\hline & CAA191 & Portugal & Ferula communis & KX871846 & KX871802 & KX871711 \\
\hline & CAA195 & Portugal & Ferula communis & KX871847 & KX871803 & KX871712 \\
\hline & CAA197 & Portugal & Ferula communis & KX871848 & KX871804 & KX871713 \\
\hline & CAA202 & Portugal & Melia azedarach & KX871849 & KX871805 & KX871714 \\
\hline & CAA231 & Portugal & Hydrangea macrophylla & KX871850 & KX871806 & KX871715 \\
\hline & CAA233 & Portugal & Hydrangea macrophylla & KX871851 & KX871807 & KX871716 \\
\hline & CAA242 & Portugal & Hydrangea macrophylla & KX871852 & KX871808 & KX871717 \\
\hline & CAA319 & Portugal & Eucalyptus globulus & KT440900 & KT440960 & KX871718 \\
\hline & CAA320 & Portugal & Eucalyptus globulus & KT440901 & KT440961 & KX871719 \\
\hline & CAA326 & Portugal & Pyracantha coccinea & KX871853 & KX871809 & KX871720 \\
\hline & CAA327 & Portugal & Pyracantha coccinea & KX871854 & KX871810 & KX871721 \\
\hline & CAA332 & Portugal & Eucalyptus globulus & KT440902 & KT440962 & KX871722 \\
\hline & CAA341 & Portugal & Eucalyptus globulus & KT440903 & KT440963 & KX871723 \\
\hline & CAA344 & Portugal & Eucalyptus globulus & KT440904 & KT440964 & KX871724 \\
\hline & CAA351 & Portugal & Eucalyptus globulus & KT440905 & KT440965 & KX871725 \\
\hline & CAA357 & Portugal & Eucalyptus globulus & KT440906 & KT440966 & KX871726 \\
\hline & CAA359 & Portugal & Eucalyptus globulus & KT440907 & KT440967 & KX871727 \\
\hline & CAA392 & Portugal & Quercus robur & KX871855 & KX871811 & KX871728 \\
\hline & CAA398 & Portugal & Eucalyptus globulus & KX871856 & KX871812 & KX871729 \\
\hline & CAA400 & Portugal & Eucalyptus globulus & KT440908 & KT440968 & KX871730 \\
\hline & CAA401 & Portugal & Eucalyptus globulus & KT440909 & KT440969 & KX871731 \\
\hline & CAA406 & Portugal & Eucalyptus globulus & KT440910 & KT440970 & KX871732 \\
\hline & CAA420 & Portugal & Eucalyptus globulus & KT440911 & KT440971 & KX871733 \\
\hline & CAA427 & Portugal & Eucalyptus globulus & KT440912 & KT440972 & KX871734 \\
\hline
\end{tabular}




\begin{tabular}{|c|c|c|c|c|c|c|}
\hline \multirow[b]{2}{*}{ Species } & \multirow[b]{2}{*}{ Isolate $^{\mathrm{a}}$} & \multirow[b]{2}{*}{ Origin } & \multirow[b]{2}{*}{ Host } & \multicolumn{3}{|c|}{ GenBank Accession $^{b}$} \\
\hline & & & & ITS & tef1 & $t u b 2$ \\
\hline & CAA434 & Portugal & Eucalyptus globulus & KT440913 & KT440973 & KX505927 \\
\hline & CAA441 & Portugal & Eucalyptus globulus & KT440914 & KT440974 & KX871735 \\
\hline & CAA455 & Portugal & Eucalyptus globulus & KT440915 & KT440975 & KX505928 \\
\hline & CAA464 & Portugal & Eucalyptus globulus & KT440916 & KT440976 & KX871736 \\
\hline & CAA466 & Portugal & Eucalyptus globulus & KT440917 & KT440977 & KX871737 \\
\hline & CAA468 & Portugal & Olea europaea & KX871857 & KX871813 & KX871738 \\
\hline & CAA475 & Portugal & Olea europaea & KX871858 & KX871814 & KX871739 \\
\hline & CAA546 & Portugal & Eucalyptus globulus & KT440918 & KT440978 & KX871740 \\
\hline & CAA549 & Portugal & Eucalyptus globulus & KT440919 & KT440979 & KX871741 \\
\hline & CAA550 & Portugal & Eucalyptus globulus & KX871859 & KX871815 & KX871742 \\
\hline & CAA571 & Portugal & Eucalyptus globulus & KX871860 & KX871816 & KX871743 \\
\hline & CAA647 & Portugal & Eucalyptus globulus & KT440920 & KT440980 & KX871744 \\
\hline & CAA648 & Portugal & Eucalyptus globulus & KT440921 & KT440981 & KX871745 \\
\hline & CAA649 & Portugal & Eucalyptus globulus & KX871861 & KX871817 & KX871746 \\
\hline & CAA723 & Portugal & Tilia platyphyllos & KX871862 & KX871818 & KX871747 \\
\hline & CAA741 & Portugal & Acacia longifolia & KX871863 & KX871819 & KX871748 \\
\hline & CAA743 & Portugal & Acacia longifolia & KX871864 & KX871820 & KX871749 \\
\hline & CAA747 & Portugal & Acacia longifolia & KX871865 & KX871821 & KX871750 \\
\hline & CAA749 & Portugal & Acacia longifolia & KX871866 & KX871822 & KX871751 \\
\hline & CAA750 & Portugal & Acacia longifolia & KX871867 & KX871823 & KX871752 \\
\hline & CAA751 & Portugal & Acacia longifolia & KX871868 & KX871824 & KX871753 \\
\hline \multirow[t]{2}{*}{ N. batangarum } & CBS124924 & Cameroon & Terminalia catappa & FJ900607 & FJ900653 & FJ900634 \\
\hline & CBS124923 & Cameroon & Terminalia catappa & FJ900608 & FJ900654 & FJ900635 \\
\hline \multirow{2}{*}{ N. brasiliense } & CMM1338 & Brazil & Mangifera indica & JX513630 & $J X 513610$ & KC794031 \\
\hline & CMM1285 & Brazil & Mangifera indica & $J X 513628$ & $J X 513608$ & KC794030 \\
\hline \multirow{2}{*}{ N. cordaticola } & CBS123634 & South Africa & Syzygium cordatum & EU821898 & EU821868 & EU821838 \\
\hline & CBS123635 & South Africa & Syzygium cordatum & EU821903 & EU821873 & EU821843 \\
\hline \multirow[t]{2}{*}{ N. cryptoaustrale } & CMW23785 & South Africa & Eucalyptus sp. & FJ752742 & FJ752713 & FJ752756 \\
\hline & CMW20738 & South Africa & Eucalyptus citriodora & FJ752740 & FJ752710 & FJ752754 \\
\hline \multirow[t]{2}{*}{ N. eucalypticola } & CBS115679 & Australia & Eucalyptus grandis & AY615141 & AY615133 & AY615125 \\
\hline & CBS115766 & Australia & Eucalyptus rossi & AY615143 & AY615135 & AY615127 \\
\hline \multirow[t]{8}{*}{ N. eucalyptorum } & CBS115791 & South Africa & Eucalyptus grandis & $A F 283686$ & $A Y 236891$ & AY236920 \\
\hline & CAA369 & Portugal & Eucalyptus globulus & KT440922 & KT440982 & KX871773 \\
\hline & CAA450 & Portugal & Eucalyptus globulus & KT440923 & KT440983 & KX871774 \\
\hline & CAA517 & Portugal & Eucalyptus globulus & KT440924 & KT440984 & KX871775 \\
\hline & CAA518 & Portugal & Eucalyptus globulus & KX871883 & KX871839 & KX871776 \\
\hline & CAA520 & Portugal & Eucalyptus globulus & KT440925 & KT440985 & KX871777 \\
\hline & CAA522 & Portugal & Eucalyptus globulus & KT440926 & KT440986 & KX871778 \\
\hline & CAA528 & Portugal & Eucalyptus globulus & KT440927 & KT440987 & KX871779 \\
\hline
\end{tabular}




\begin{tabular}{|c|c|c|c|c|c|c|}
\hline \multirow[b]{2}{*}{ Species } & \multirow[b]{2}{*}{ Isolate $^{\mathrm{a}}$} & \multirow[b]{2}{*}{ Origin } & \multirow[b]{2}{*}{ Host } & \multicolumn{3}{|c|}{ GenBank Accession $^{b}$} \\
\hline & & & & ITS & tef1 & $t u b 2$ \\
\hline & CAA532 & Portugal & Eucalyptus globulus & KT440928 & KT440988 & KX871780 \\
\hline & CAA535 & Portugal & Eucalyptus globulus & KT440929 & KT440989 & KX871781 \\
\hline & CAA536 & Portugal & Eucalyptus globulus & KT440930 & KT440990 & KX871782 \\
\hline & CAA539 & Portugal & Eucalyptus globulus & KX871884 & KX871840 & KX871783 \\
\hline & CAA542 & Portugal & Eucalyptus globulus & KT440931 & KT440991 & KX871784 \\
\hline & CAA558 & Portugal & Eucalyptus globulus & KT440932 & KT440992 & KX871785 \\
\hline & CAA561 & Portugal & Fraxinus excelsior & KX871885 & KX871841 & KX871786 \\
\hline & CAA601 & Portugal & Eucalyptus globulus & KT440933 & KT440993 & KX871787 \\
\hline & CAA604 & Portugal & Eucalyptus globulus & KT440934 & KT440994 & KX871788 \\
\hline & CAA618 & Portugal & Eucalyptus globulus & KT440935 & KT440995 & KX871789 \\
\hline & CAA624 & Portugal & Eucalyptus globulus & KT440936 & KT440996 & KX871790 \\
\hline & CAA651 & Portugal & Eucalyptus globulus & KT440937 & KT440997 & KX871791 \\
\hline & CAA680 & Portugal & Eucalyptus globulus & KT440938 & KT440998 & KX871792 \\
\hline & CAA683 & Portugal & Eucalyptus globulus & KT440939 & KT440999 & KX871793 \\
\hline & CAA695 & Portugal & Eucalyptus globulus & KT440940 & KT441000 & KX871794 \\
\hline & CAA709 & Portugal & Eucalyptus globulus & KT440941 & KT441001 & KX505920 \\
\hline & CAA712 & Portugal & Eucalyptus globulus & KT440942 & KT441002 & KX871795 \\
\hline & CAA713 & Portugal & Eucalyptus globulus & KT440943 & KT441003 & KX505921 \\
\hline & CAA714 & Portugal & Eucalyptus globulus & KX871886 & KX871842 & KX871796 \\
\hline & PE20 & Portugal & Eucalyptus globulus & KT440944 & KT441004 & KX871797 \\
\hline & PE21 & Portugal & Eucalyptus globulus & KT440945 & KT441005 & KX871798 \\
\hline & PE23 & Portugal & Eucalyptus globulus & KX871887 & KX871843 & KX871799 \\
\hline \multirow[t]{2}{*}{ N. hellenicum } & CERC1947 & Greece & Pistacia vera & $K P 217053$ & KP217061 & KP217069 \\
\hline & CERC1948 & Greece & Pistacia vera & KP217054 & $K P 217062$ & KP217070 \\
\hline \multirow[t]{3}{*}{ N. kwambonambiense } & CBS123639 & South Africa & Syzygium cordatum & EU821900 & EU821870 & EU821840 \\
\hline & CBS123641 & South Africa & Syzygium cordatum & EU821919 & EU821889 & EU821859 \\
\hline & CAA755 & Portugal & Eucalyptus globulus & KT440946 & KT441006 & KX505917 \\
\hline \multirow[t]{12}{*}{ N. luteum } & CBS110299 & Portugal & Vitis vinifera & $A Y 259091$ & AY573217 & $D Q 458848$ \\
\hline & CBS110497 & Portugal & Vitis vinifera & EU673311 & EU673277 & EU673092 \\
\hline & CAA200 & Portugal & Melia azedarach & KX871869 & KX871825 & KX871754 \\
\hline & CAA203 & Portugal & Melia azedarach & KX871870 & KX871826 & KX871755 \\
\hline & CAA352 & Portugal & Quercus robur & KX871871 & KX871827 & KX871756 \\
\hline & CAA360 & Portugal & Fraxinus ornus & KX871872 & KX871828 & KX871757 \\
\hline & CAA362 & Portugal & Fraxinus ornus & KX871873 & KX871829 & KX871758 \\
\hline & CAA365 & Portugal & Quercus robur & KX871874 & KX871830 & KX871759 \\
\hline & CAA379 & Portugal & Melia azedarach & KX871875 & KX871831 & KX871760 \\
\hline & CAA412 & Portugal & Populus alba & KX871876 & KX871832 & KX871761 \\
\hline & CAA505 & Portugal & Fraxinus ornus & KX871877 & KX871833 & KX871762 \\
\hline & CAA628 & Portugal & Fraxinus excelsior & KX505911 & KX505902 & KX505929 \\
\hline
\end{tabular}




\begin{tabular}{|c|c|c|c|c|c|c|}
\hline \multirow[b]{2}{*}{ Species } & \multirow[b]{2}{*}{ Isolate $^{\mathrm{a}}$} & \multirow[b]{2}{*}{ Origin } & \multirow[b]{2}{*}{ Host } & \multicolumn{3}{|c|}{ GenBank Accession $^{b}$} \\
\hline & & & & ITS & tef1 & tub2 \\
\hline & CAA720 & Portugal & Tilia platyphyllos & KX871878 & KX871834 & KX871763 \\
\hline \multirow{2}{*}{ N. macroclavatum } & CBS118223 & Australia & Eucalyptus globulus & $D Q 093196$ & $D Q 093217$ & $D Q 093206$ \\
\hline & WAC12445 & Australia & Eucalyptus globulus & $\widetilde{D Q 093197}$ & $\widetilde{D Q 093218}$ & $D Q 093208$ \\
\hline \multirow{2}{*}{ N. mangiferae } & CBS118531 & Australia & Mangifera indica & AY 615185 & DQ093221 & AY 615172 \\
\hline & CBS118532 & Australia & Mangifera indica & AY615186 & DQ093220 & AY615173 \\
\hline \multirow[t]{2}{*}{ N. mediterraneum } & CBS121718 & Greece & Eucalyptus sp. & GU251176 & GU251308 & GU251836 \\
\hline & CBS121558 & USA & Vitis vinifera & GU799463 & GU799462 & GU799461 \\
\hline N. nonquaesitum & CBS126655 & USA & Umbellularia californica & GU251163 & GU251295 & GU251823 \\
\hline N. nonquaesitum & PD301 & Chile & Vaccinium corymbosum & GU251164 & GU251296 & GU251824 \\
\hline \multirow[t]{2}{*}{ N. occulatum } & CBS128008 & Australia & Eucalyptus grandis hybrid & EU301030 & EU339509 & EU339472 \\
\hline & MUCC286 & Australia & Eucalyptus pellita & EU736947 & EU339511 & EU339474 \\
\hline \multirow{12}{*}{ N. parvum } & CMW9081 & New Zealand & Populus nigra & $A Y 236943$ & AY236888 & $A Y 236917$ \\
\hline & UCR-NP2 & USA & Vitis vinifera & AORE01001444 & AORE01000046 & AORE01001255 \\
\hline & CBS110301 & Portugal & Vitis vinifera & AY259098 & AY573221 & EU673095 \\
\hline & CAA189 & Portugal & Ferula communis & KX871879 & KX871835 & KX871766 \\
\hline & CAA192 & Portugal & Ferula communis & KX505905 & KX505892 & KX505913 \\
\hline & CAA384 & Portugal & Rosa sp. & KX871880 & KX871836 & KX871767 \\
\hline & CAA386 & Portugal & Rosa sp. & KX871881 & KX871837 & KX871768 \\
\hline & CAA608 & Portugal & Aesculus hippocastanum & KX871882 & KX871838 & KX871769 \\
\hline & CAA692 & Portugal & Eucalyptus globulus & KT440950 & KT441010 & KX871770 \\
\hline & CAA704 & Portugal & Eucalyptus globulus & KT440947 & KT441007 & KX505914 \\
\hline & PE17 & Portugal & Eucalyptus globulus & KT440948 & KT441008 & KX871771 \\
\hline & PE18 & Portugal & Eucalyptus globulus & KT440949 & KT441009 & KX871772 \\
\hline N. pennatisporum & MUCC510 & Australia & Allocasuarina fraseriana & EF591925 & EF591976 & EF591959 \\
\hline N. protearum & MUCC497 & Australia & Santalum acuminatum & EF591912 & EF591965 & EF591948 \\
\hline \multirow{2}{*}{ N. ribis } & CBS115475 & USA & Ribes sp. & $A Y 236935$ & $A Y 236877$ & AY236906 \\
\hline & CBS121.26 & Unknown & Ribes rubrum & $A F 241177$ & AY236879 & AY236908 \\
\hline \multirow[t]{2}{*}{ N. umdonicola } & CBS123645 & South Africa & Syzygium cordatum & EU821904 & EU821874 & EU821844 \\
\hline & CBS123646 & South Africa & Syzygium cordatum & EU821905 & EU821875 & EU821845 \\
\hline \multirow[t]{3}{*}{ N. vitifusiforme } & $5 \mathrm{H} 022$ & California & Juglans regia & KF778869 & KF779059 & KF778964 \\
\hline & B8 & Italy & Vitis vinifera & KC469638 & KX505897 & KX505922 \\
\hline & B9 & Italy & Vitis vinifera & KX505908 & KX505898 & KX505923 \\
\hline
\end{tabular}

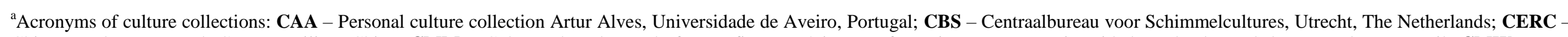

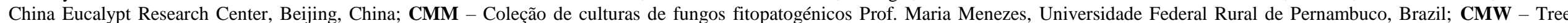

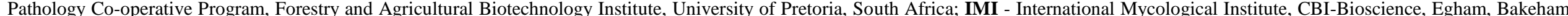

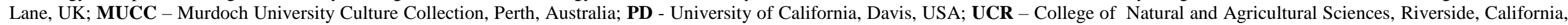
USA; WAC - Department of Agriculture, Western Australia Plant Pathogen Collection, South Perth, Western Australia.

${ }^{\mathrm{b}} \mathrm{S}$ Sequence numbers in italics were retrieved from GenBank. All others were determined in the present study.

Isolates in bold are ex-type cultures. 


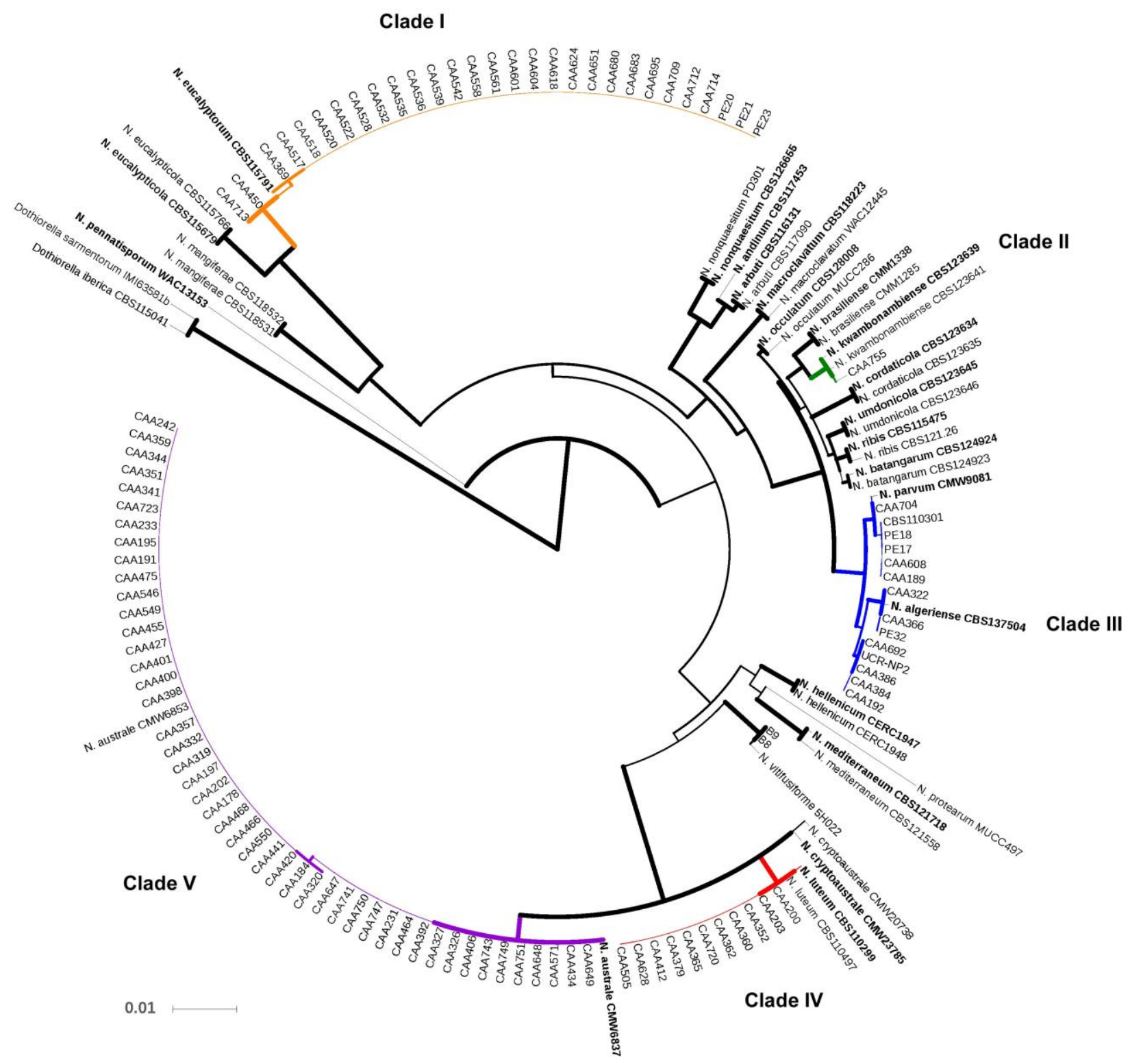

Fig. 2 - Combined ITS, tefl and tub2 maximum likelihood tree based on the Tamura 3-parameter model. A discrete Gamma distribution was used to model evolutionary rate differences among sites. The tree is drawn to scale, with branch lengths measured in the number of substitutions per site. The thickness of branches is proportional to bootstrap support values.

\section{Molecular Characterization}

BOX-PCR fingerprinting analysis divided the 351 isolates into 7 distinct clusters, which were presumed to represent distinct species. A total of 99 isolates representative of each group were selected for further molecular characterization (Table 1). The 7 clusters formed by the BOX-PCR fingerprinting analysis were resolved into 5 clades by multilocus (ITS, tef1 and tub2) phylogenetic analysis (Fig. 2).

Clades I, II, IV and V were clearly resolved and represent the species $N$. eucalyptorum, $N$. kwambonambiense, $N$. luteum and $N$. australe respectively. Clade III contained isolates belonging to two species ( $N$. parvum and $N$. algeriense) and was further divided into 3 subclades. However, these showed incongruence between phylogenetic analyses results obtained from each individual locus (data 
not shown) and from the combined dataset (Fig. 2). Moreover, there were no fixed alleles between the different subclades.

Table 2 Neofusicoccum species isolated in this study and their respective hosts

\begin{tabular}{|c|c|}
\hline Species & Host \\
\hline \multirow[t]{13}{*}{ N. australe } & Acacia longifolia $^{\mathrm{a}, \mathrm{b}}$ \\
\hline & Castanea sativa $a^{\mathrm{a}, \mathrm{b}}$ \\
\hline & Eucalyptus globulus \\
\hline & Ferula $_{\text {communis }}^{\mathrm{a}, \mathrm{b}}$ \\
\hline & Fraxinus excelsior $^{\mathrm{a}, \mathrm{b}}$ \\
\hline & Hydrangea macrophylla $a^{\mathrm{a}, \mathrm{b}}$ \\
\hline & Melia azedarach ${ }^{\mathrm{a}, \mathrm{b}}$ \\
\hline & Olea europaea ${ }^{\mathrm{b}}$ \\
\hline & Populus alba ${ }^{\mathrm{a}, \mathrm{b}}$ \\
\hline & Pyracantha coccinea $^{\mathrm{a}, \mathrm{b}}$ \\
\hline & Quercus robur \\
\hline & Tilia platyphyllos ${ }^{\mathrm{a}, \mathrm{b}}$ \\
\hline & Ulmus minor $^{\mathrm{a}, \mathrm{b}}$ \\
\hline \multirow[t]{2}{*}{ N. eucalyptorum } & Eucalyptus globulus \\
\hline & Fraxinus excelsior ${ }^{\mathrm{a}, \mathrm{b}}$ \\
\hline N. kwambonambiense & Eucalyptus globulus \\
\hline \multirow[t]{7}{*}{ N. luteum } & Fraxinus excelsior $^{\mathrm{a}, \mathrm{b}}$ \\
\hline & Fraxinus ornus $^{\mathrm{a}, \mathrm{b}}$ \\
\hline & Melia azedarach ${ }^{\mathrm{a}, \mathrm{b}}$ \\
\hline & Populus alba \\
\hline & Populus tremula ${ }^{\mathrm{a}, \mathrm{b}}$ \\
\hline & Quercus robur \\
\hline & Tilia platyphyllos ${ }^{\mathrm{a}, \mathrm{b}}$ \\
\hline \multirow[t]{6}{*}{ N. parvum } & Aesculus hippocastanum ${ }^{\mathrm{b}}$ \\
\hline & Eucalyptus globulus \\
\hline & Ferula communis ${ }^{\mathrm{a}, \mathrm{b}}$ \\
\hline & Malus domestica \\
\hline & Melia azedarach ${ }^{\mathrm{a}, \mathrm{b}}$ \\
\hline & Rosa sp. ${ }^{\mathrm{a}, \mathrm{b}}$ \\
\hline
\end{tabular}

${ }^{a}$ new host reported for the species

${ }^{\mathrm{b}}$ first report from Portugal

Within the clades formed by $N$. eucalyptorum and $N$. australe two subclades were also noticeable. However, a comparison of sequences of the three loci from members of each subclade showed minor differences between them. Thus, only 1 bp difference in the tefl of $N$. eucalyptorum isolates and $1 \mathrm{bp}$ in the tub2 sequence of $N$. australe isolates.

\section{Discussion}

In this study a collection of 351 isolates retrieved from a large diversity of plant hosts was characterised by morphological and PCR typing analysis. Selected representative isolates of each PCR 
typing group were further characterised by multilocus phylogenetic analyses. The isolates studied grouped into five clades, four of which clearly represented distinct species (Fig. 2).

The clade containing $N$. parvum and $N$. algeriense (Clade III) was not clearly resolved, exhibiting incongruence between phylogenetic analysis results obtained from each individual locus and the combined dataset. A similar inconsistency was seen in phylogenetic analyses based on MAT genes (Lopes et al. 2016). By applying the principle of Phylogenetic Species Recognition (Taylor et al. 2000) where the transition from concordance to conflict determines the limits of species Lopes et al. (2016) considered that this clade represented a single species and synonymized $N$. algeriense with $N$. parvum. This study is in agreement with this previous finding.

Neofusicoccum australe and $N$. eucalyptorum were the most common species found. Neofusicoccum australe was originally regarded as native to Australia but since then it has been shown to have a widespread distribution occurring on a broad range of hosts (Sakalidis et al. 2011, Phillips et al. 2013). In Portugal, N. australe was found on Rubus sp. (Phillips et al. 2006), Quercus robur (Barradas et al. 2013), Eucalyptus globulus (Barradas et al. 2016), Robinia pseudoacacia (van Niekerk et al. 2004) and several species of conifers (Alves et al. 2013). To our knowledge, this study is the first to report $N$. australe occurring on A. longifolia, C. sativa, F. communis, F. excelsior, H. macrophylla, $M$. azedarach, $P$. alba, $P$. coccinea, $T$. platyphyllos and $U$. minor. It is also the first time that this species is found on O. europaea in Portugal. Another interesting finding was the isolation of $N$. australe from A. longifolia, being the first report of this species colonizing Acacia spp. outside of Australia. This could have serious repercussions on the dissemination of $N$. australe in Portugal since Acacia spp. are introduced exotic species that have spread rapidly to several new areas, from the coast to inland forests. Colonization of the invasive species A. longifolia will allow N. australe to be rapidly introduced into new geographic areas, possibly infecting new hosts.

Neofusicoccum eucalyptorum was first found on diseased Eucalyptus grandis and E. nitens in South Africa (Smith et al. 2001). Later, the species was isolated from cankers on native and planted eucalypts in eastern Australia (Slippers et al. 2004). Based on the dominance and wide distribution in eastern Australia, the authors suggested that the pathogen is probably native to this area (Slippers et al. 2004). Meanwhile, the presence of $N$. eucalyptorum was also detected on eucalypt species in other countries including Portugal (Barradas et al. 2016). Several authors suggested that the occurrence of the species on Eucalyptus in others parts of the world is a consequence of anthropogenic actions due to the large amounts of germplasm traded (Pérez et al. 2009, Barradas et al. 2016). Although this species is apparently specialized in the infection of Eucalyptus spp., it has also been associated with other genera in the Myrtaceae (Pérez et al. 2009, Pérez et al. 2010). In our study we report the occurrence of $N$. eucalyptorum in Fraxinus excelsior (Oleaceae) planted as ornamental. This is the first time that $N$. eucalyptorum is associated with a host outside of the family Myrtaceae. However, it is important to note that the $F$. excelsior tree from which the fungus was isolated was surrounded by a large number of eucalypts. Thus, it is possible that $F$. excelsior was colonized due to the high pressure of the surrounding inoculum or the fungus used it as a transition host. Further studies should be carried out to test the pathogenicity of $N$. eucalyptorum to this host and evaluate the impact that host jumps may have on the fungus host expansion and pathogenicity.

The species $N$. luteum and $N$. parvum were also found in this study although the number of isolates was lower. Both species are known to occur on a wide range of hosts worldwide (Phillips et al. 2013). Neofusicoccum luteum has been associated with dieback and canker mostly on crops (e.g. Phillips 2002, Úrbez-Torres et al. 2013) but also on ornamentals (Marincowitz et al. 2008, Varela et al. 2011). In Portugal, N. luteum has been found to infect conifers (Alves et al. 2013), Quercus robur (Barradas et al. 2013), grapevines, Fraxinus angustifolia and Sophora japonica (Phillips et al. 2002). In our study we found new host associations namely with $M$. azedarach, F. ornus, F. excelsior, $P$. alba, P. tremula and T. platyphyllos, all of them planted as ornamentals. 
Neofusicoccum parvum is probably the species within the genus with the widest geographic distribution, host range and proven ability to cause disease (Phillips et al. 2013, Sakalidis et al. 2013). It has been found associated with many forest species (Iturritxa et al. 2011), fruit trees (Ismail et al. 2013) and ornamental plants (Marincowitz et al. 2008, Zlatković et al. 2016). In Portugal, N. parvum was found associated with Protea cynaroides and P. repens (Crous et al. 2013), grapevines (Phillips 2002), conifers (Alves et al. 2013) and E. globulus (Barradas et al. 2016). To our knowledge, this study is the first to report the association of $N$. parvum with Rosa spp., F. communis, M. azedarach and also the first occurrence of $N$. parvum on A. hippocastanum in Portugal. The fungus was only recently associated for the first time with A. hippocastanum in the Western Balkans, showing symptoms of canker and dieback (Zlatković et al. 2016). In our study it was isolated from severely affected trees with trunk cankers (Fig. 1) and planted as ornamentals on streetscapes. However, since no pathogenicity tests were carried out we cannot conclude that $N$. parvum was the cause of the observed symptoms. This aspect should be addressed in future studies.

The presence, in this study, of species in such a wide diversity of hosts confirms that Neofusicoccum species are opportunistic fungi that can potentially colonize most plant hosts that it comes into contact with and represents a threat to vulnerable plants. This study reinforces the urgent need to understand the routes of introduction and dissemination of these fungi, not only in natural environments but also in the less studied urban environments where many potential hosts are planted as ornamentals.

\section{Acknowledgements}

This work was financed by European Funds through COMPETE and National Funds through the Portuguese Foundation for Science and Technology (FCT) to project ALIEN (PTDC/AGRPRO/2183/2014 - POCI-01-0145-FEDER-016788), CESAM (UID/AMB/50017/2013 - POCI-010145-FEDER-007638), Artur Alves (FCT Investigator Programme - IF/00835/2013), Anabela Lopes (PhD grant - SFRH/BD/85615/2012) and Carla Barradas (PhD grant - SFRH/BD/77939/2011).

\section{References}

Alves A, Barradas C, Phillips AJL, Correia A. 2013 - Diversity of Botryosphaeriaceae species associated with conifers in Portugal. European Journal of Plant Pathology 135, 791-804.

Alves A, Correia A, Luque J, Phillips AJL. 2004 - Botryosphaeria corticola sp. nov. on Quercus species, with notes and description of Botryosphaeria stevensii and its anamorph Diplodia mutila. Mycologia 96, 598-613.

Alves A, Crous PW, Correia A, Phillips AJL. 2008 - Morphological and molecular data reveal cryptic speciation in Lasiodiplodia theobromae. Fungal Diversity 28, 1-13.

Alves A, Phillips AJL, Henriques I, Correia A. 2007 - Rapid differentiation of species of Botryosphaeriaceae by PCR fingerprinting. Research in Microbiology 158, 112-121.

Barradas C, Correia A, Alves A. 2013 - First Report of Neofusicoccum australe and Neofusicoccum luteum Associated with Canker and Dieback of Quercus robur in Portugal. Plant Disease 97, 560.

Barradas C, Phillips AJL, Correia A, Diogo E, Bragança H, Alves A. 2016 - Diversity and potential impact of Botryosphaeriaceae species associated with Eucalyptus globulus plantations in Portugal. European Journal of Plant Pathology 146, 245-257.

Berraf-Tebbal A, Guerreiro MA, Phillips AJL. 2014 - Phylogeny of Neofusicoccum species associated with grapevine trunk diseases in Algeria, with description of Neofusicoccum algeriense sp. nov. Phytopathologia Mediterranea 53, 416-427. 
Burgess TI, Barber PA, Hardy GEStJ. 2005 - Botryosphaeria spp. associated with eucalypts in Western Australia, including the description of Fusicoccum macroclavatum sp. nov. Australasian Plant Pathology 34, 557-567.

Crous PW, Denman S, Taylor JE, Swart L, Bezuidenhout CM, Hoffman L, Palm ME, Groenewald JZ. 2013 - Cultivation and Disease of Proteaceae: Leucadendron, Leucospermum, and Protea: Second Edition. CBS Biodiversity Series.

Crous PW, Slippers B, Wingfield MJ, Rheeder J, Marasas WFO, Phillips AJL, Alves A, Burgess T, Barber P, Groenewald JZ. 2006 - Phylogenetic lineages in the Botryosphaeriaceae. Studies in Mycology 55, 235-253.

Glass NL, Donaldson G. 1995 - Development of primer sets designed for use with PCR to amplify conserved genes from filamentous ascomycetes. Applied Environmental Microbiology 61, 1323-1330.

Golzar H, Burgess TI. 2011 - Neofusicoccum parvum, a causal agent associated with cankers and decline of Norfolk Island pine in Australia. Australasian Plant Pathology 40, 484-489.

Hall TA. 1999 - BioEdit: a user-friendly biological sequence alignment editor and analysis program for Windows 95/98/NT. Nucleic Acids Symposium Series 41, 95-98.

ICNF. 2013 - IFN6 - Áreas dos usos do solo e das espécies florestais de Portugal continental. Resultados preliminares. [pdf], 34 pp. Instituto da Conservação da Natureza e das Florestas. Lisboa (accessed April 2016).

Inderbitzin P, Bostock RM, Trouillas FP, Michailides TJ. 2010 - A six locus phylogeny reveals high species diversity in Botryosphaeriaceae from California almond. Mycologia 102, 1350-1368.

Ismail AM, Cirvilleri G, Lombard L, Crous PW, Groenewald JZ, Polizzi G. 2013 - Characterization of Neofusicoccum species causing mango dieback in Italy. Journal of Plant Pathology 95, 549557.

Iturritxa E, Slippers B, Mesanza N, Wingfield MJ. 2011 - First report of Neofusicoccum parvum causing canker and die-back of Eucalyptus in Spain. Australasian Plant Disease Notes 6, 5759.

Letunic I, Bork P. 2016 - Interactive tree of life (iTOL) v3: an online tool for the display and annotation of phylogenetic and other trees. Nucleic Acids Research 44, W242-245.

Lopes A, Phillips AJL, Alves A. 2016 - Mating type genes in the genus Neofusicoccum: mating strategies and usefulness in species delimitation. Fungal Biology, In Press doi: 10.1016/j.funbio.2016.08.011

Marincowitz S, Groenewald JZ, Wingfield MJ, Crous PW. 2008 - Species of Botryosphaeriaceae occurring on Proteaceae. Persoonia 21, 111-118.

McDonald V, Eskalen A. 2011 - Botryosphaeriaceae Species Associated with Avocado Branch Cankers in California. Plant Disease 95, 1465-1473.

Mondello V, Picolo SL, Conigliaro G, Alfonzo A, Torta L, Burruano S. 2013 - First report of Neofusiccoccum vitifusiforme and presence of other Botryosphaeriaceae species associated with Botryosphaeria dieback of grapevine in Sicily (Italy). Phytopathologia Mediterranea 52, 388-396.

Ngobisa AICN, Abidin MAZ, Wong MY, Noordin MWDW. 2013 - Neofusicoccum ribis Associated with Leaf Blight on Rubber (Hevea brasiliensis) in Peninsular Malaysia. The Plant Pathology Journal 29, 10-16.

O'Donnell K, Cigelnik E. 1997 - Two divergent intragenomic rDNA ITS2 types within a monophyletic lineage of the fungus Fusarium are nonorthologous. Molecular and Phylogenetics Evolution 7, $103-116$. 
Pavlic D, Slippers B, Coutinho TA, Wingfield MJ. 2009a - Molecular and phenotypic characterization of three phylogenetic species discovered within the Neofusicoccum parvum/N. ribis complex. Mycologia 101, 636-647.

Pavlic D, Slippers B, Coutinho TA, Wingfield MJ. 2009b - Multiple gene genealogies and phenotypic data reveal cryptic species of the Botryosphaeriaceae: A case study on the Neofusicoccum parvum/N. ribis complex. Molecular Phylogenetics and Evolution 51, 259-268.

Pérez CA, Wingfield MJ, Slippers B, Altier NA, Blanchette RA. 2010 - Endophytic and cankerassociated Botryosphaeriaceae occurring on non-native Eucalyptus and native Myrtaceae trees in Uruguay. Fungal Diversity 41, 53-69.

Pérez CA, Wingfield MJ, Slippers B, Altierd NA, Blanchette RA. 2009 - Neofusicoccum eucalyptorum, a Eucalyptus pathogen, on native Myrtaceae in Uruguay. Plant Pathology 58, 964-970.

Pérez SF, Meriño-Gergichevich C, Guerrero JC. 2014 - Detection of Neofusicoccum nonquaesitum causing dieback and canker in highbush blueberry from Southern Chile. Journal of Soil Science and Plant Nutrition 14, 581-588.

Phillips AJL, Alves A, Abdollahzadeh J, Slippers B, Wingfield MJ, Groenewald JZ, Crous PW. 2013 The Botryosphaeriaceae: genera and species known from culture. Studies in Mycology 76, 51167.

Phillips AJL, Alves A, Correia A, Luque J, 2005. Two new species of Botryosphaeria with brown, 1septate ascospores and Dothiorella anamorphs. Mycologia 97, 513-529.

Phillips AJL, Oudemans PV, Correia A, Alves A. 2006 - Characterisation and epitypification of Botryosphaeria corticis, the cause of blueberry cane canker. Fungal Diversity 21, 141-155.

Phillips AJL, Fonseca F, Povoa V, Castilho R, Nolasco G, 2002 - A reassessment of the anamorphic fungus Fusicoccum luteum and description of its teleomorph Botryosphaeria lutea sp. nov. Sydowia 54, 59-77.

Phillips AJL. 2002 - Botryosphaeria species associated with diseases of grapevines in Portugal. Phytopathologia Mediterranea 41, 3-18.

Sakalidis ML, Hardy GEStJ, Burgess TI. 2011 - Class III endophytes, clandestine movement amongst hosts and habitats and their potential for disease; a focus on Neofusicoccum australe. Australasian Plant Pathology 40, 510-521.

Sakalidis ML, Slippers B, Wingfield BD, Hardy GE St J, Burguess TI. 2013 - The challenge of understanding the origin, pathways and extent of fungal invasions: global populations of the Neofusicoccum parvum - N. ribis species complex. Diversity and Distributions 19, 873-883.

Slippers B, Boissin E, Phillips AJL, Groenewald JZ, Lombard L, Wingfield MJ, Postma A, Burgess T, Crous PW. 2013 - Phylogenetic lineages in the Botryosphaeriales: a systematic and evolutionary framework. Studies in Mycology 76, 31-49.

Slippers B, Fourie G, Crous PW, Coutinho TA, Wingfield BD, Carnegie AJ, Wingfield MJ. 2004 Speciation and distribution of Botryosphaeria spp. on native and introduced Eucalyptus trees in Australia and South Africa. Studies in Mycology 50, 343-58.

Slippers B, Summerell BA, Crous PW, Coutinho TA, Wingfield BD, Wingfield MJ. 2005 Preliminary studies on Botryosphaeria species from Southern Hemisphere conifers in Australasia and South Africa. Australasian Plant Pathology 34, 213-220.

Slippers B, Wingfield MJ. 2007 - Botryosphaeriaceae as endophytes and latent pathogens of woody plants: diversity, ecology and impact. Fungal Biology Reviews 21, 90-106.

Smith H, Crous PW, Wingfield MJ, Coutinho TA, Wingfield BD. 2001 - Botryosphaeria eucalyptorum sp. nov., a new species in the B. dothidea-complex on Eucalyptus in South Africa. Mycologia 93, 277-285. 
Tamura K, Stecher G, Peterson D, Filipski A, Kumar S. 2013 - MEGA6: Molecular Evolutionary Genetics Analysis Version 6.0. Molecular Biology and Evolution 30, 2725-2729.

Taylor JW, Jacobson DJ, Kroken S, Kasuga T, Geiser DM, Hibbett DS, Fisher MC. 2000 Phylogenetic species recognition and species concepts in fungi. Fungal Genetics and Biology 31, 21-32.

Thomidis T, Michailides TJ, Exadaktylou E. 2011 - Neofusicoccum parvum associated with fruit rot and shoot blight of peaches in Greece. European Journal of Plant Pathology 131, 661-668.

Thompson JD, Gibson TJ, Plewniak F, Jeanmougin F, and Higgins DG. 1997 - The ClustalX windows interface: flexible strategies for multiple sequence alignment aided by quality analysis tools. Nucleic Acids Research 25, 4876-4882.

Triki MA, HadjTaieb SK, Cheffi M, Gharbi Y, Rhouma A. 2015 - First Report of Dieback of Olive Trees caused by Neofusicoccum australe in Tunisia. Journal of Plant Pathology 97, 209-220.

Úrbez-Torres JR, Peduto F, Vossen PM, Krueger WH, Gubler WD. 2013 - Olive twig and branch dieback: etiology, incidence and distribution in California. Plant Disease 97, 231-244.

van Niekerk JM, Crous PW, Groenewald JZE, Fourie PH, Halleen F. 2004 - DNA phylogeny, morphology and pathogenicity of Botryosphaeria species on grapevines. Mycologia 96, 781798.

Varela CP, Fernández VR, Vásquez JPM, Casal OA. 2011 - First Report of Dieback on Hybrid Rhododendrons Caused by Neofusicoccum luteum and N. parvum in Spain. Plant Disease 95, 221.

White TJ, Bruns T, Lee S, Taylor J, 1990 - Amplification and direct sequencing of fungal genes for phylogenies. In: Innis MA, Gelfand DH, Sninsky JJ, White TJ, (eds) PCR protocols: a guide to methods and applications. Academic Press, California, 315-322.

Yu Z, Tang G, Peng S, Chen H, Zhai M. 2015 - Neofusicoccum parvum causing canker of seedlings of Juglans regia in China. Journal of Forestry Research 26, 1019-1024.

Zlatković M, Keča N, Wingfield MJ, Jami F, Slippers B. 2016 - Botryosphaeriaceae associated with the die-back of ornamental trees in the Western Balkans. Antonie van Leeuwenhoek 109, 543564. 